\title{
CHRONIC INTESTINAL CONSTIPATION OF ATYPICAL ETIOLOGY IN PRESCHOOL CHILD
}

\author{
Camila Fachin ${ }^{1}$, Carlos Riedi ${ }^{2}$, Tarick Salim Ali ${ }^{1}$, Milena Conceição ${ }^{1}$, Miguel Angelo \\ Agulham $^{1}$, and Andre Dias ${ }^{1}$ \\ ${ }^{1}$ Federal University of Parana \\ ${ }^{2}$ Federal University Parana
}

June 17,2020

\begin{abstract}
There is a large spectrum of abnormalities in the CFTR gene that leads to a partial function of the chloride channel and, thereby, an uncommon presentation of the disease, the "CFTR-related diseases". This report aims to present a case of a patient with a single gene mutation related to cystic fibrosis, with an unusual clinical presentation among CFTR related diseases chronic intestinal constipation. This patient is not a cystic fibrosis patient because he does not fulfil the disease's classic criteria. Furthermore, since the mutation found is not yet described in the literature, it could be related to CFTR-related disease.
\end{abstract}

\section{KEYWORDS:}

Cystic Fibrosis

CFTR related disease

Chronic constipation

\section{ABBREVIATIONS:}

CFTR: Cystic fibrosis transmembrane conductance regulator

G: Guanine

HC: Hospital de Clínicas

Leu: Leucine

Phe: Phenilalanine

T: Tiamine

This research did not receive any specific grant from funding agencies in the public, commercial, or not-forprofit sectors.

\section{INTRODUCTION:}

Cystic fibrosis is a recessive genetic disease caused by mutation in the two alleles of the gene CFTR. It can be diagnosed by classic symptoms and laboratory tests, such as sweat testing or genetic testing for the identification of CFTR mutations [1]. When performing the genetic test, the most frequent mutations for certain ethnic groups are investigated [2]. There is a large spectrum of abnormalities in the CFTR gene that leads to a partial function of the chloride canal and, thereby, an uncommon presentation of the disease. These presentations do not fully meet the diagnostic criteria for cystic fibrosis and are therefore classified 
as "CFTR-related diseases" [3]. This article aims to report a case of a patient with a single gene mutation related to cystic fibrosis, with an unusual clinical presentation among CFTR related diseases.

\section{CASE REPORT:}

A 4-year-old male admitted at the University Hospital with intestinal constipation since birth, remaining up to 7 days without defecation followed by abdominal distension and soiling. After the first year of life, there was a worsening in the clinical scenario with an increase in the number of days without bowel movements. At another service, the patient had performed anoplasty and several sessions of anal dilatation. On physical examination, the abdomen was slightly distended, painless and without palpable masses. In rectal examination, presence of a large amount of softened stool. Initially, it was prescribed to the patient: dietary orientation, rectal enemas with sorbitol and sodium lauryl sulfate solution and polyethylene glycol orally. After 2 months of treatment, the patient returns with partial improvement of the condition, but maintaining soiling and a palpable fecaloma. The patient was then hospitalized and submitted to rectal enema with lactated ringer and $12 \%$ glycerin solution. The fecaloma was manually removed under general anesthesia. Barium enema confirmed megarectum. Surgical rectal biopsy was performed and no changes were observed in ganglion cells In addition, anorectal manometry was normal. Due to the persistence of fecaloma formation, a double-barrel colostomy was performed. Due to the persistence of fecaloma, even with the colostomy, cystic fibrosis was investigated. The sweat test was borderline and a therapeutic trial with acetylcysteine and pancrelipase was initiated; besides performing the genetic test for cystic fibrosis. The test identified the variation p.Leu1156Phe $\mathrm{G}>\mathrm{T}$, a variant of the CFTR gene of undetermined meaning.

\section{DISCUSSION:}

The diagnostic criteria for cystic fibrosis suggest that there must be both the characteristic clinical phenotype of the disease and also a documented dysfunction of the CFTR protein [1]. The patient of this report cannot be considered a cystic fibrosis patient because he does not have the classic clinical features of the disease, the sweat chloride test has shown indeterminate values and he doesn't present two known genes or gene variant that causes the disease.

The CFTR-related diseases are those that have association with CFTR gene dysfunction, but do not fulfil the diagnostic criteria for the disease [4]. This new classification is related to the advances in genetic testing that guide the diagnosis criteria for cystic fibrosis and this new group of diseases. In fact, it is still difficult to distinguish those two entities [3], and as new discoveries are made, new classifications systems will be adopted.

Three clinical entities have strong support in the literature, being defined as CFTR-related diseases: bilateral congenital absence of the vas deferens with CFTR dysfunction, recurrent acute pancreatitis or chronic pancreatitis with CFTR dysfunction, and disseminated bronchiectasis with CFTR dysfunction [3]. In addition to these presentations, other phenotypes were observed associated with mutations in the CFTR gene, such as sinusitis and bronchopulmonary allergic aspergillosis [2]. Many of the patients with these phenotypes will never progress to a phenotype of frank cystic fibrosis and, therefore, their follow-up may be done differently from that usually done in patients with cystic fibrosis [3].

The patient reported has a p.Leu1156Phe $\mathrm{G}>\mathrm{T}$ alteration, in which is observed a guanine to thymine change at nucleotide 3599 in exon 18, which would lead to a replacement of leucine by phenylalanine in the protein sequence at residue 1156 (L1156F) [5]. Although this change is localized in the CFTR gene, it still represents an undetermined meaning mutation [5]. Given the presentation of the reported patient, oligosymptomatic cystic fibrosis with only chronic constipation, indeterminate sweat chloride test and a genetic alteration in the CFTR coding area, without the characterization of frank cystic fibrosis, it is believed that the mutation found, not yet described in the literature, could be related and could be classified as a CFTR-related disease. For more accurate diagnosis, in addition to the sweat test and mutation investigation in the CFTR gene, measurements of nasal potential difference [2] [3] and intestinal current measurement [3] should be performed.

\section{REFERENCES:}


Rosenstein BJ, Cutting GR, Boat TF, Cantin A, Dorkin HL, Durie P, FitzSimmons S, Knowles MR, Saiman L, Tullis E: The diagnosis of cystic fibrosis: a consensus statement. J Pediatr 1998, 132:589-595.

Peadar G Noone; Michael R Knowles: Review 'CFTR-opathies': disease phenotypes associated with cystic fibrosis transmembrane regulator gene mutations. Respiratory Research Vol 2 No 6. Available online http://respiratory-research.com/content/ 2/6/328.

C. Bombieri et al. Recommendations for the classification of diseases as CFTRrelated disorders. Journal of Cystic Fibrosis Volume 10 Suppl 2 (2011) S86-S102

Molecular Genetics Laboratory, Hospital for Sick Children. Cystic Fibrosis and CFTR- Related Disorders. OMG1620P/02. Available from: URL:

https://www.sickkids.ca/PDFs/Paediatric\%20Laboratory\%20Medicine/info-sheets/

56255-CF_OMG1620P_02.pdf

Cystic Fibrosis Mutation Database. Mutation Details for c.3468G>T. Available from: URL: http://www.genet.sickkids.on.ca/cftr/MutationDetailPage.external?sp=1640

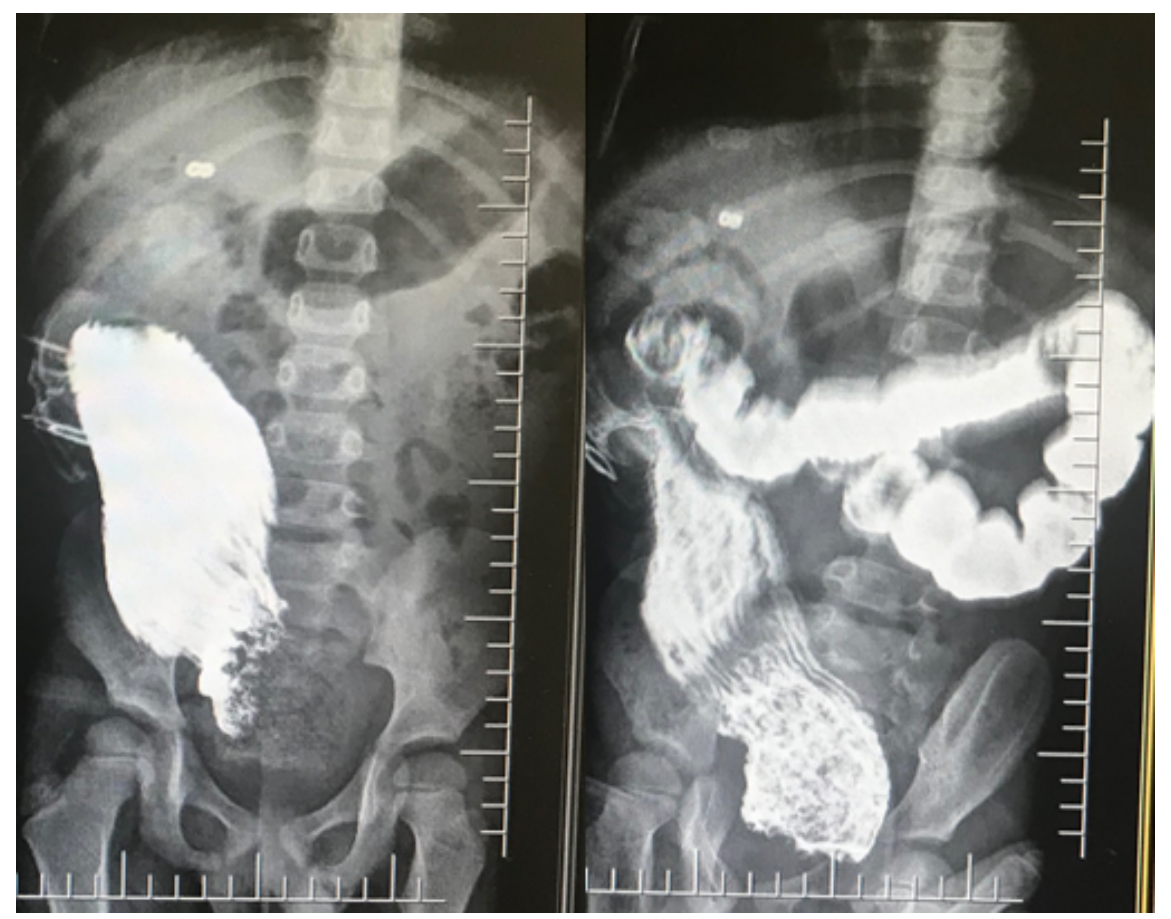




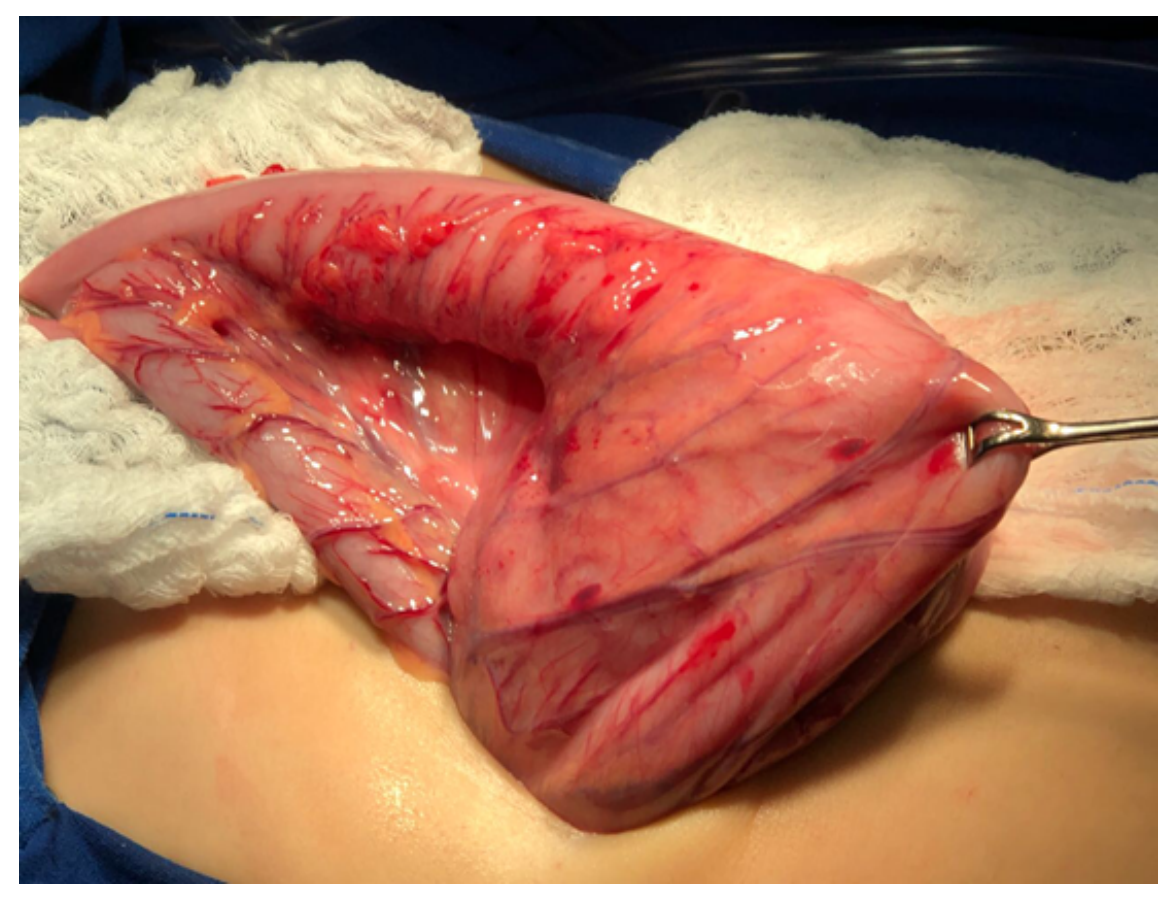

